\title{
Modelling, simulation and experiment of the spherical flexible joint stiffness
}

\author{
Songyu Li ${ }^{1}$, Liquan Wang ${ }^{1}$, Shaoming Yao ${ }^{2}$, Peng Jia ${ }^{1}$, Feihong Yun ${ }^{1}$, Wenxue Jin ${ }^{1}$, and Dong Lv $^{1}$ \\ ${ }^{1}$ College of Mechanical and Electronical Engineering, Harbin Engineering University, Harbin, 150001, China \\ ${ }^{2}$ AMRC with Boeing, the University of Sheffield, Sheffield, S60 5TZ, UK \\ Correspondence: Liquan Wang (wangliquan@hrbeu.edu.cn)
}

Received: 11 September 2017 - Revised: 5 January 2018 - Accepted: 1 February 2018 - Published: 19 February 2018

\begin{abstract}
The spherical flexible joint is extensively used in engineering. It is designed to provide flexibility in rotation while bearing vertical compression load. The linear rotational stiffness of the flexible joint is formulated. The rotational stiffness of the bonded rubber layer is related to inner radius, thickness and two edge angles. FEM is used to verify the analytical solution and analyze the stiffness. The Mooney-Rivlin, Neo Hooke and Yeoh constitutive models are used in the simulation. The experiment is taken to obtain the material coefficient and validate the analytical and FEM results. The Yeoh model can reflect the deformation trend more accurately, but the error in the nearly linear district is bigger than the Mooney-Rivlin model. The Mooney-Rivlin model can fit the test result very well and the analytical solution can also be used when the rubber deformation in the flexible joint is small. The increase of Poisson's ratio of the rubber layers will enhance the vertical compression stiffness but barely have effect on the rotational stiffness.
\end{abstract}

\section{Introduction}

The spherical flexible joints are widely used as non-rigid connection in aerospace and offshore oil and gas industry. The flexible joint is the critical part of the flexible bearing nozzle in the solid rocket boosters (SRB). It allows nozzle to deflect in given directions for booster thrust vector control (Kumar et al., 2015). Elastomeric bearings are flexible joints used in helicopter rotor hubs. These bearings are spherical hinges to withstand centrifugal and lateral forces while tolerating rotational, flap and lead-lag motions (Donguy, 2015). In the offshore oil and gas industry, the flex joints, also known as FlexJoints ${ }^{\circledR}$, are used to connect TLP (tension leg platform) to the subsea foundation (Kumar, 2000). A typical flexible joint consists of alternately laminated spherical rubber and metal layers. For different application, the configuration of the metal layers may be slightly different. The metal layers of rocket solid booster flexible bearings may protrude outside the rubber layers to resist to higher temperature (Lampani et al., 2012). On the contrary, to protect the metal from seawater corrosion, the metal layers of FlexJoints ${ }^{\circledR}$ are wrapped in the rubber layer (Gunderson et al., 1992).
Due to near incompressibility of rubber, the bearing load capacity and stiffness will increase dramatically when the lateral edge motion of the rubber is constrained. Meanwhile the relatively low shear modulus may facilitate the rotation and release the bending moment produced stress at the connection. Therefore, the stiffness is one of the most important factors in the flexible joint design. Typically, the vertical compression stiffness is designed sufficiently high to bear compression loading and the rotational stiffness needs to be relatively low. The effect factors of both stiffnesses are necessary to be analysed. Moreover the stiffness also plays a part in failure analysis (Stevenson and Harris, 1992). Thus an analytical solution of the stiffness is necessary. The effective compression modulus is defined to formulate the vertical compression stiffness (Tsai, 2012). Gent and Lindley (1959) proposed an approximation to calculate the effective compression modulus of the bonded rubber. That method was called "mean pressure" method. The solution are obtained by superposing two stages. In the first stage, the elastomer is compressed between unbonded rigid plates; in the second stage, the shear stresses are then applied to the bonded surface. This approximation was used for rubber discs and infinite long rubber 


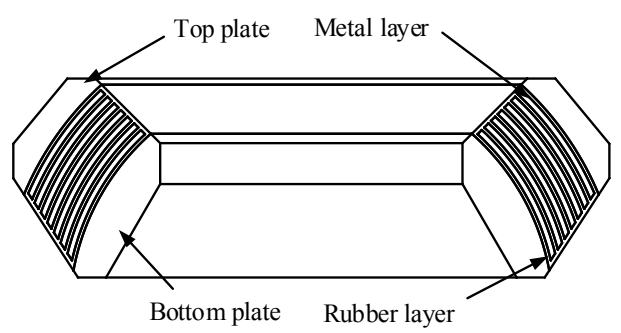

Figure 1. Typical flexible joint.

strips based on two kinematic assumptions and one stress assumption:

a. planes parallel to the rigid surface will keep plane and parallel during or after deformation;

b. lines normal to the rigid surface will be changed to parabolic after deformation produced by the compression;

c. normal stress components in three directions are equal to the mean pressure.

The rubber block in this approximation is assumed incompressible, which will overestimate the compression stiffness when the shape factor is high. Therefore, another method was proposed with regard of bulk compressibility (Chalhoub and Kelly, 1990, 1991; Kelly, 1993). The method applies to the rubber layers of circular, infinite-strip and square shapes with Poisson' ratio between 0.49 and 0.5. Tsai and Pai proposed a new method for the full range of Poisson's ratio with two kinematic assumptions to calculate the effective compression moduli of the rubber layers with infinite-strip, circular and square shapes (Tsai and Lee, 1998). Wang et al. (2017) used the same method to calculate the effective compression modulus of the spherical bonded rubber layer.

The flexible joint undergoes pure shear deformation under the torsional moment. Some studies have been taken on the shear deformation of the rubber bearings with circular and square shapes (He et al., 2012; Mishra and Igarashi, 2013). Exact closed-form expressions are derived for the torsional stiffness of the spherical rubber bush mountings (Horton and Tupholme, 2005). Zhang et al. (2012) used nonlinear FEM to simulate the SRB flexible joint structural behaviour and conducted experiments to validate the analysis. But the stiffness was not studied. Chen and Yang (2015) proposed an analytical method to calculate the compression and torsional stiffness of the helicopter rotor elastomeric bearings with incompressible assumption. An experiment was conducted to validate the analytical solution. But the calculation error is too big.

The linear vertical stiffness of the spherical bonded rubber layer has been presented in the previous work (Wang et al., 2017). A closed-form expression of the linear rotational stiffness of the bonded rubber layer is proposed. The influence

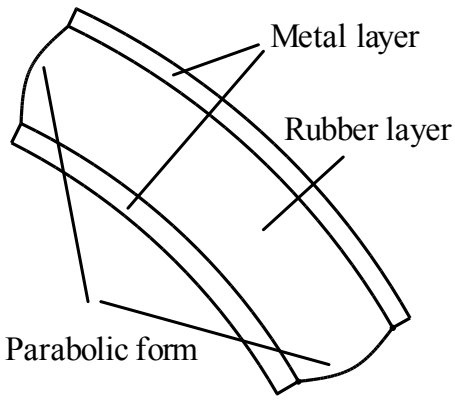

Figure 2. The parabolic form of rubber boundary.

factors on the rotational stiffness are studied. FEM is used to verify the analytical result and analyse the stiffness of the flexible joint. The experiment is taken to validate the simulation and analytical results.

\section{Formulation of the linear stiffness}

The sectional view of a typical flexible joint is shown in Fig. 1. It is spherical and consists of laminated rubber and metal layers. The top plate is fixed and loads are applied to the bottom plate. Generally, the spherical rubber and metal layers are equivalent to be in series and have a coincident centre. Thus the total linear stiffness, $K$, of the flexible joint could be calculated by the expression below:

$K=\left(\sum \frac{1}{K_{i}}\right)^{-1}$

where $K_{i}$ is the vertical compression stiffness (or rotational stiffness) of the rubber layer $i$.

Some assumptions are given to calculate the linear vertical compression stiffness and rotational stiffness of the flexible joint:

a. the metal layer is rigid;

b. planes parallel to the metal layer will remain plane and parallel during or after compression deformation;

c. lines normal to the metal layer will be changed to parabolic after the deformation produced by the compression loads, as shown in Fig. 2.

\subsection{Vertical compression stiffness}

Figure 3 shows the cross section of a single spherical rubber layer. According to Wang et al. (2017), the vertical stiffness can be expressed as 


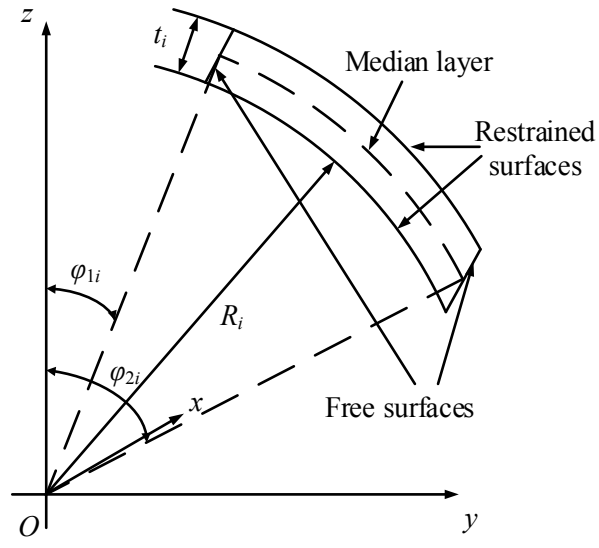

Figure 3. A single rubber layer of the flexible joint.

$$
\begin{gathered}
K_{\mathrm{c}}=\left[4 \pi(R+t / 2)^{2} G\left(x_{1}-x_{2}\right)-\frac{2 \pi(R+t / 2)^{2} G v}{k \varepsilon_{\mathrm{c}}(1-2 v)}\right. \\
\left.\int_{x_{1}}^{x_{2}} C_{1} P_{d}(x)+C_{2} Q_{d}(x)+k\left(\varepsilon_{\mathrm{c}}-\delta \varepsilon_{\mathrm{c}}\right) d x\right] \frac{\cos ^{2} \varphi_{\mathrm{e}}}{t},
\end{gathered}
$$

where $G$ and $v$ are respectively the initial shear modulus and Poisson's ratio of the rubber; $R$ and $t$ are respectively the inner radius and thickness of the rubber layer; $k$ is the volume modulus, $\varepsilon_{\mathrm{c}}$ is the effective compression strain; $P_{d}(x)$ and $Q_{d}(x)$ are respectively Legendre function of the 1 st and 2 nd kind; $d=\frac{1}{2} \sqrt{1+2 \alpha \frac{1-2 v}{1-v}}-\frac{1}{2}$ is the degree of the function; $\delta \varepsilon_{\mathrm{c}}=-\frac{t(3 R+2 t) \varepsilon_{\mathrm{c}}}{2\left(3 R^{2}+3 R t+t^{2}\right)} ; x_{1}=\cot \varphi_{1}, x_{2}=\cot \varphi_{2} ; \varphi_{e}=\left(\varphi_{1}+\right.$ $\left.\varphi_{2}\right) / 2$.

$$
\begin{aligned}
& C_{1}= \frac{C_{3}}{C_{4}} \frac{k \alpha}{4\left(x_{1}^{2}-1\right)\left(x_{2}^{2}-1\right)} \frac{\delta-v}{1-v} \varepsilon_{\mathrm{c}}, \\
& C_{2}=\left\{\begin{array}{c}
\left.-C_{1}\left\{\frac{\alpha P_{d}\left(x_{1}\right)}{2}+\frac{x_{1}(d+1)\left[x_{1} P_{d}\left(x_{1}\right)-P_{d+1}\left(x_{1}\right)\right]}{x_{1}^{2}-1}\right\}\right\} \\
+\frac{k}{2}\left[\frac{1-2 v}{1-v}(1-\delta)+2 \delta-1\right] \varepsilon_{\mathrm{c}}
\end{array}\right\} \\
& C_{3}=\left(x_{2}^{2}-1\right)\left[\alpha\left(x_{1}^{2}-1\right)+2 x_{1}^{2}(d+1)\right] Q_{d}\left(x_{1}\right) \\
&-\left(x_{1}^{2}-1\right)\left[\alpha\left(x_{2}^{2}-1\right)+2 x_{2}^{2}(d+1)\right] Q_{d}\left(x_{2}\right) \\
&+2(d+1)\left[\left(x_{1}^{2}-1\right) x_{2} Q_{d+1}\left(x_{2}\right)-\left(x_{2}^{2}-1\right) x_{1} Q_{d+1}\left(x_{1}\right)\right],
\end{aligned}
$$

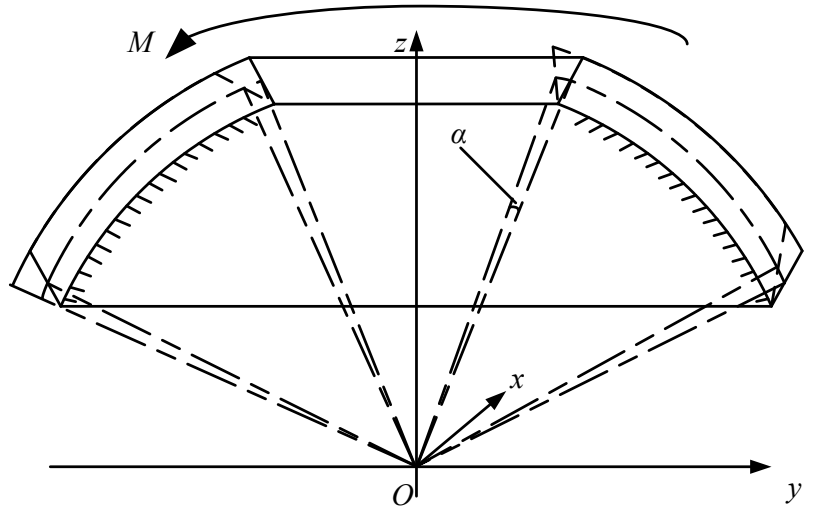

Figure 4. The boundary conditions and loading of rotational rubber layer.

$$
\begin{aligned}
C_{4} & =\left\{\frac{\alpha Q_{d}\left(x_{1}\right)}{2}+\frac{x_{1}(d+1)\left[x_{1} Q_{d}\left(x_{1}\right)-Q_{d+1}\left(x_{1}\right)\right]}{x_{1}^{2}-1}\right\} \\
& \cdot\left\{\frac{\alpha P_{d}\left(x_{2}\right)}{2}+\frac{x_{2}(d+1)\left[x_{2} P_{d}\left(x_{2}\right)-P_{d+1}\left(x_{2}\right)\right]}{x_{2}^{2}-1}\right\} \\
& -\left\{\frac{\alpha P_{d}\left(x_{1}\right)}{2}+\frac{x_{1}(d+1)\left[x_{1} P_{d}\left(x_{1}\right)-P_{d+1}\left(x_{1}\right)\right]}{x_{1}^{2}-1}\right\} \\
& \cdot\left\{\frac{\alpha Q_{d}\left(x_{2}\right)}{2}+\frac{x_{2}(d+1)\left[x_{2} Q_{d}\left(x_{2}\right)-Q_{d+1}\left(x_{2}\right)\right]}{x_{2}^{2}-1}\right\} \\
\alpha & =1 /\left[\frac{R(R+t)}{t(2 R+t)} \ln \frac{R+t}{R}-\frac{1}{2}\right] .
\end{aligned}
$$

\subsection{Rotational stiffness}

Because the rubber layer is axisymmetric about $z$ axis, the rotational stiffness about any axes which pass through the origin in $x y$ plane will be the same. The linear rotational stiffness about the $x$ axis is calculated by semi-inverse method. As shown in Fig. 4, the bottom is fixed and a torsional moment, $M$, is applied to the top surface. An angle, $\alpha$, around $x$ axis is produced in the rubber layer. Only shear deformation occur when the thickness is much smaller than the inner radius, so there is no deformation along $r$ direction and $u(r)=0$. Any point in the rubber layer moves along a circle of radius $\left(y^{2}+z^{2}\right)^{0.5}$ centered on the $x$ axis and the displacement parallel to $x$ axis is zero. In that case, for any point on $z y$ plane $\left(\theta=90^{\circ}\right)$, the displacement in the $\varphi$ direction only related to $r$ and $u_{\varphi}=V_{1}(r)$, the displacement in the $\theta$ direction is zero and $u_{\theta}=0$; for any point on the $x z$ plane $(\theta=0)$, the displacement in the $\varphi$ direction is zero and $u_{\varphi}=0$; for any point on the $z$ axis $(\theta=\varphi=0)$, the displacement in the $\theta$ direction is $V_{2}(r)$; for any point on the $x$ axis $(\theta=0$ and $\varphi=90^{\circ}$ ), the displacement in the $\theta$ direction is zero. To meet 
the above conditions, the displacement components are assumed as below:

$u_{r}=0$,

$u_{\varphi}=V(r) \sin \theta$,

$u_{\theta}=V(r) \cos \varphi \cos \theta$,

where $V(r)$ is the function of $r$. Because Poisson's ratio has a low influence on the rotational stiffness, the rubber is assumed as completely incompressible here. Thus the volume modulus is infinite and $\varepsilon_{r r}+\varepsilon_{\varphi \varphi}+\varepsilon_{\theta \theta}=0$. According to the displacement assumption, the strain in the rubber layer can be calculated as

$\varepsilon_{r r}=\varepsilon_{\varphi \varphi}=\varepsilon_{\theta \theta}=\varepsilon_{\theta \varphi}=\varepsilon_{\varphi \theta}=0$,

$\varepsilon_{r \varphi}=\varepsilon_{\varphi r}=\frac{1}{2}\left(\frac{d V(r)}{d r}-\frac{V(r)}{r}\right) \sin \theta$,

$\varepsilon_{r \theta}=\varepsilon_{\theta r}=\frac{1}{2}\left(\frac{d V(r)}{d r}-\frac{V(r)}{r}\right) \cos \theta \cos \varphi$.

According to the Hooke law, the stress is

$\sigma_{r r}=\sigma_{\varphi \varphi}=\sigma_{\theta \theta}$,

$\sigma_{r \varphi}=\sigma_{\varphi r}=G\left(\frac{d V(r)}{d r}-\frac{V(r)}{r}\right) \sin \theta$,

$\sigma_{r \theta}=\sigma_{\theta r}=G\left(\frac{d V(r)}{d r}-\frac{V(r)}{r}\right) \cos \theta \cos \varphi$,

$\sigma_{\theta \varphi}=\sigma_{\varphi \theta}=0$.

Thus the equilibrium equations of the rubber layer in spherical coordinate system (Landau and Lifshitz, 1986) can be expressed as

$\frac{\partial \sigma_{\varphi \varphi}}{\partial r}=0$,

$\frac{\partial \sigma_{\varphi \varphi}}{\partial \theta}+G\left(r \frac{d^{2} V}{d r^{2}}+2 \frac{d V}{d r}-2 \frac{V}{r}\right) \sin \varphi \cos \varphi \cos \theta=0$,

$\frac{\partial \sigma_{\varphi \varphi}}{\partial \varphi}+G\left(r \frac{d^{2} V}{d r^{2}}+2 \frac{d V}{d r}-2 \frac{V}{r}\right) \sin \varphi=0$.

According to the first equation in Eq. (6), $\sigma_{\varphi \varphi}$ is not related to $r . r \frac{d^{2} V}{d r^{2}}+2 \frac{d V}{d r}-2 \frac{V}{r}$ needs to be 0 if the latter two equations in Eq. (6) validate for any $\varphi$ and $\theta$. Then the latter two equations become $\partial \sigma_{\varphi \varphi} / \partial \theta=0$ and $\partial \sigma_{\varphi \varphi} / \partial \varphi=0$. So, the general solution of the equations is

$\sigma_{\varphi \varphi}=C_{1}$,

$V(r)=C_{2} r+C_{3} / r^{2}$,

where $C_{1}, C_{2}, C_{3}$ are constants.

To make the results more general, define a parameter $r=R+s, s \in[0, t]$. The boundary condition on the free surfaces of the rubber layer can be approximately expressed as $\sigma_{\varphi \varphi}=\sigma_{\theta \theta}=\sigma_{r r}=C_{1}=0$ when $\varphi=\varphi_{1}$ and $\varphi=\varphi_{2}$. Substitute Eq. (7) into Eq. (5) and yield

$\sigma_{r \varphi}=\sigma_{\varphi r}=-\frac{3 G C_{3}}{(R+s)^{3}} \sin \theta$,

$\sigma_{r \theta}=\sigma_{\theta r}=-\frac{3 G C_{3}}{(R+s)^{3}} \cos \theta \cos \varphi$.

The torsional moment $M$ can be expressed by the stress integration as

$$
\begin{aligned}
M= & -\int_{0}^{2 \pi} \int_{\varphi_{1}}^{\varphi_{2}}\left[(R+s) \sin \varphi \sin \theta\left(-\sin \varphi \sigma_{\varphi r}\right)\right. \\
& \left.-(R+s) \cos \varphi\left(\cos \varphi \sin \theta \sigma_{\varphi r}+\cos \theta \sigma_{\theta r}\right)\right] \\
& (R+s)^{2} \sin \varphi d \varphi d \theta .
\end{aligned}
$$

Substitute Eq. (8) into Eq. (9) and yield

$$
C_{3}=-\frac{M}{\pi G\left[\left(3 \cos \varphi_{1}+\cos ^{3} \varphi_{1}\right)-\left(3 \cos \varphi_{2}+\cos ^{3} \varphi_{2}\right)\right]} .
$$

The boundary condition on the restrained surfaces of the rubber layer can be expressed as

$u_{\theta}=u_{\varphi}=0, s=0$,

$$
\begin{gathered}
\left(u_{\varphi} \cos \varphi \sin \theta+u_{\theta} \cos \theta\right) \frac{(R+t) \cos \varphi}{D}+\left(u_{\varphi} \sin \varphi\right) \\
\frac{(R+t) \sin \theta \sin \varphi}{D}=\alpha D, s=t,
\end{gathered}
$$

where $D=(R+t) \sqrt{\sin ^{2} \theta \sin ^{2} \varphi+\cos ^{2} \varphi}$, which is the distance between a point on the top restrained surface and $x$ axis.

Substitute Eqs. (3) and (7) into Eq. (12) and yield

$C_{2}=-\frac{C_{3}}{R^{3}}=\frac{(R+t)^{3}}{(R+t)^{3}-R^{3}} \alpha$.

Thus the rotational stiffness $K_{\mathrm{r} i}$ of the rubber layer $i$ is

$$
\begin{aligned}
K_{\mathrm{r} i} & =\frac{M_{i}}{\alpha_{i}}=\pi G \frac{R_{i}^{3}\left(R_{i}+t_{i}\right)^{3}}{\left(R_{i}+t_{i}\right)^{3}-R_{i}^{3}}\left[\left(3 \cos \varphi_{1 i}+\cos ^{3} \varphi_{1 i}\right)\right. \\
& \left.-\left(3 \cos \varphi_{2 i}+\cos ^{3} \varphi_{2 i}\right)\right] .
\end{aligned}
$$

Because the rubber thickness $t_{i}$ is far less than the rubber layer radius $R_{i}$, define $\rho_{i}=t_{i} / R_{i}$ and $\rho_{i} \ll 0$. Then Eq. (14) will be simplified as

$$
\begin{aligned}
K_{i} & \approx \pi G \frac{R_{i}^{6}\left(1+3 \rho_{i}\right)}{R_{i}^{3}\left(3 \rho_{i}\right)}\left[\begin{array}{c}
\left(3 \cos \varphi_{1 i}+\cos ^{3} \varphi_{1 i}\right) \\
-\left(3 \cos \varphi_{2 i}+\cos ^{3} \varphi_{2 i}\right)
\end{array}\right] \\
& =\pi G R_{i}^{3}\left(\frac{1}{3 \rho_{i}}+1\right)\left[\begin{array}{c}
\left(3 \cos \varphi_{1 i}+\cos ^{3} \varphi_{1 i}\right) \\
-\left(3 \cos \varphi_{2 i}+\cos ^{3} \varphi_{2 i}\right)
\end{array}\right] .
\end{aligned}
$$




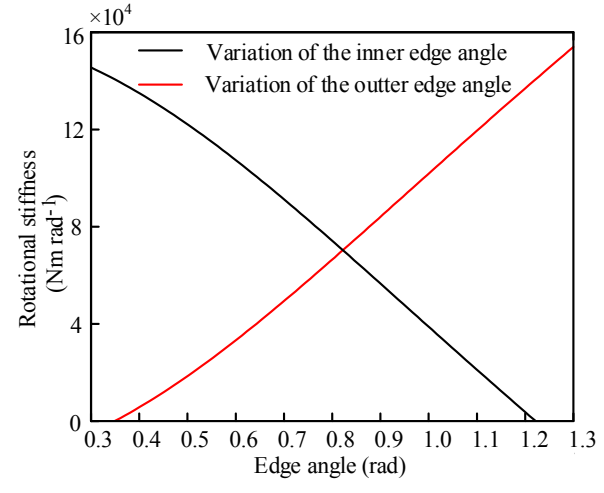

Figure 5. Rotational stiffness variation along with edge angles.

\section{Rotational stiffness analyses}

The vertical compression stiffness of the bonded rubber layer has been analyzed in Wang et al. (2017), here the rotational stiffness will be analyzed. According to Eqs. (5) and (7), the rubber layer bears pure shear stress in rotation and the rotational stiffness is proportional to the shear modulus. Besides the shear modulus, the rotational stiffness is also related to the inner radius, the thickness and two edge angles. A bonded rubber layer is modelled to analyze the rotational stiffness by individual variables. Its inner radius, $R$, is $100 \mathrm{~mm}$; the thickness, $t$, is $2 \mathrm{~mm}$; the edge angles of free surfaces, $\varphi_{1}$ and $\varphi_{2}$, are, respectively, 20 and $70^{\circ}$. Because the rubber thickness is far less than the radius, which is the displacement assumption, the analytical result will be inaccurate when the thickness-radius ratio is rather low.

Figure 5 shows the relation between the rotational stiffness and the edge angles. When the outer edge angle is constant the rotational stiffness decreases if the inner edge angle increases and when the inner angle is constant it increases if the outer edge angle increases. The trend is approximately linear when edge angles are between 0.3 and $1.3 \mathrm{rad}$.

Figure $6 \mathrm{a}$ shows the relation between the rotational stiffness and the thickness of the rubber layer. The curve is a hyperbola. When the thickness approaches to zero, the stiffness approaches to infinite. As the thickness increases from 0 to $0.5 \mathrm{~mm}$, the stiffness decreases sharply. Then the stiffness decreases gently. Figure $6 \mathrm{~b}$ shows the relation between the rotational stiffness and the inner radius of the rubber layer. It can be seen the rotational stiffness increases as the inner radius increases. Comparing Fig. $6 \mathrm{a}$ and Fig. 6b, decreasing the thickness of the rubber layer will be more effective to increase the rotational stiffness when the overall size of the rubber layer is constant.

\section{Simulation and experiment}

A prototype of the flexible joint is manufactured, as shown in Fig. 7. The flexible joint consists of four rubber layers.
Table 1. Geometric parameters of rubber layers.

\begin{tabular}{lcccc}
\hline $\begin{array}{l}\text { No. of } \\
\text { rubber } \\
\text { layer }(i)\end{array}$ & $\begin{array}{c}\text { Inner } \\
\text { radius } \\
\left(R_{i}\right) / \mathrm{mm}\end{array}$ & $\begin{array}{c}\text { Thickness } \\
\left(t_{i}\right) / \mathrm{mm}\end{array}$ & $\begin{array}{c}\text { Inner } \\
\text { edge } \\
\text { angle } \\
\left.\left(\varphi_{1 i}\right)\right)^{\circ}\end{array}$ & $\begin{array}{c}\text { Outer } \\
\text { edge } \\
\text { angle } \\
\left(\varphi_{2 i}\right) /^{\circ}\end{array}$ \\
\hline 1 & 140 & 3 & 42.4 & 74.6 \\
2 & 150 & 4 & 42.6 & 71.2 \\
3 & 161 & 4 & 42.7 & 68.2 \\
4 & 172 & 3 & 42.9 & 65.8 \\
\hline
\end{tabular}

Three metal layers are evenly arranged and fully wrapped by the rubber. The thickness of the inner and outer rubber on the edge of the flexible joint is $3 \mathrm{~mm}$. The thickness of the metal layer is $7 \mathrm{~mm}$. The specific geometric parameters of every rubber layers are shown in Table 1. A static nonlinear analysis is taken on the flexible joint in Abaqus/Standard. The stress of the flexible joint under compression is axisymmetric, so a 2-D FE model is enough for the simulation. A half 3-D joint model can be used to simulate the flexible joint under torsional moment which is symmetric about $x y$ plane.

The linear elastic material model is applied to the metal layers. The property coefficients of the metal layers are Young's modulus $E=206 \mathrm{GPa}$ and Poisson's ratio $v=0.3$. The rubber is modeled by the hyperelastic material model. The relation between stress and strain is derived from a strain energy density function. The Mooney-Rivlin, Neo Hooke and Yeoh constitutive models are used in this simulation.

The Nitrile rubber reinforced by carbon-black is used in the flexible joint prototype. Figure 8a shows the test data of the uniaxial tension test of the rubber. The test was performed on dumbbell shaped specimens prepared according to Type 1A GB/T 528-2009. The test was carried out on the tensile testing machine. The interested strain range is 0 $200 \%$ in the rubber of the flexible joint. The rubber material is nearly incompressible, which means its bulk modulus is far larger than shear modulus and Poisson's ratio is close to 0.5 . In the simulation of the rubber with tensile and shear deformation, Poisson's ratio is always assumed to be 0.5 and then bulk modulus goes to infinite. But that will overestimate the compression capacity of the rubber component when it is highly constrained and compressed. Thus the measuring of the incompressible parameter $D_{1}$ is necessary. It equals to double reciprocal of the initial volume modulus. The volumetric compression test was performed on circular cylinder specimens according to Crocker's method (Crocker and Duncan, 2001). Figure $8 \mathrm{~b}$ shows the test data of the volumetric compression test of the rubber. An approximate linear relationship is observed between pressure and volume ratio. Import the test data into Abaqus and the coefficients of Mooney-Rivlin, Neo Hooke and Yeoh constitutive models are obtained as shown in Table 2. 
Table 2. Coefficients of constitutive model.

\begin{tabular}{lllllll}
\hline Mooney-Rivlin & $C_{10}(\mathrm{MPa})$ & $C_{01}(\mathrm{MPa})$ & $D_{1}$ & - & - & - \\
& 0.260 & $9.29 \times 10^{-02}$ & $8.64 \times 10^{-03}$ & - & - & - \\
\hline \multirow{2}{*}{ Neo Hooke } & $C_{10}(\mathrm{MPa})$ & $D_{1}$ & - & - & - & - \\
& 0.239 & $2.36 \times 10^{-03}$ & - & - & - & - \\
\hline \multirow{2}{*}{ Yeoh } & $C_{10}(\mathrm{MPa})$ & $C_{20}(\mathrm{MPa})$ & $C_{30}(\mathrm{MPa})$ & $D_{1}$ & $D_{1}$ & $D_{1}$ \\
& 0.313 & -0.04 & $4.95 \times 10^{-03}$ & $1.12 \times 10^{-02}$ & $1.02 \times 10^{-05}$ & $-1.96 \times 10^{-07}$ \\
\hline
\end{tabular}
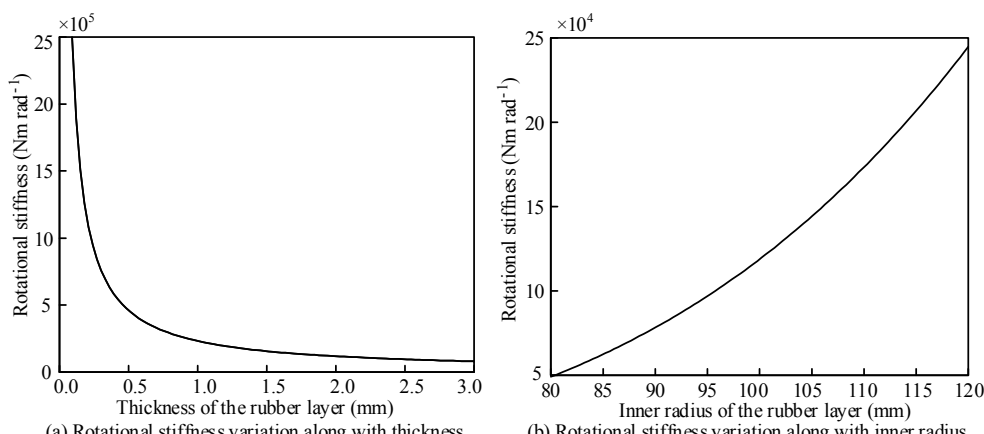

Figure 6. Rotational stiffness variation along with thickness and inner radius.
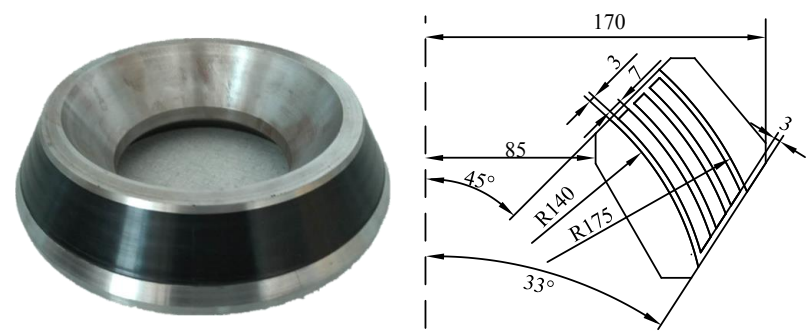

Figure 7. Detailed dimension of the flexible joint.

The increment is fixed as 0.1 in all steps in the simulation. The nlgeom option is on. As shown in Fig. 9, the top plate of the flexible joint is fixed. A reference point RP-1 is set at the coincident center. The reference point is coupled with the bottom plate of the flexible joint in the interaction module. A displacement of $2.5 \mathrm{~mm}$ in $y$ is set on the reference point in the 2-D analysis. An angle of $0.15 \mathrm{rad}$ in $z$ axis is set on the reference in both steps in the 3-D analysis. Meanwhile the symmetry boundary condition is also applied in the 3-D model.

The meshes of models are shown in Fig. 9. For nearly incompressible materials, hourglassing and volume locking phenomena may occur during simulation. Thus the hybrid and second-order elements are used to solve these problems. The 2-D axisymmetric element, CAX8R, is used for the metal part and CAX8RH is used for the rubber part in 2-D model. The 3-D solid element, C3D20R, is used for the metal part and C3D20RH is used for the rubber part in 3-D model.

To validate the theoretical and simulation results, a test device was designed to carry out the compression and rotation test for the prototype, as shown in Fig. 10. The compression and rotation loading was provided by a $2000 \mathrm{kN}$ compression tester. Four $500 \mathrm{kN}$ pressure sensors were used in parallel to measure the loading. A laser displacement sensor and an angle sensor were respectively used in compression and rotation test. In the compression test, the flexible joint was directly compressed by the pressure tester. The maximum compression loading is set as $1000 \mathrm{kN}$. The loading speed is about $40 \mathrm{kN} \mathrm{min}^{-1}$. In the rotation test, the loading was transmitted from the tester to the flexible joint by a turning stick. There are two hinges on the stick, so the pressure loading will be always perpendicular to the bottom plate of the flexible joint. The maximum rotation angle was about $8^{\circ}$. The loading speed was about $3^{\circ} \mathrm{min}^{-1}$. Both tests were repeated 5 times.

\section{Results and discussions}

Figure 11a shows the results of the compression simulation and test. It can be seen that the curve of the test data is nearly linear when the compression displacement is smaller than $1.5 \mathrm{~mm}$. Then the compression stiffness increases obviously. The result of Neo Hooke model has the most obvious difference compared with the test result. The result of MooneyRivlin model fits the test result very well in the nearly linear district. The result of Yeoh model can indicate the variation 


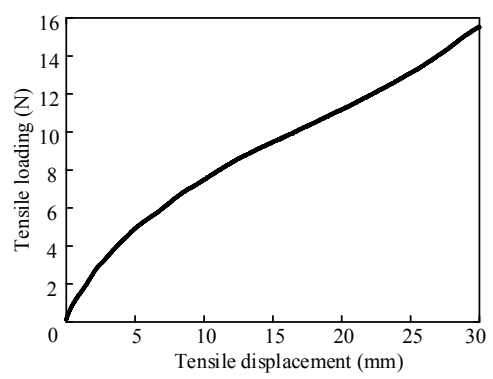

(a) Uniaxial tension test data

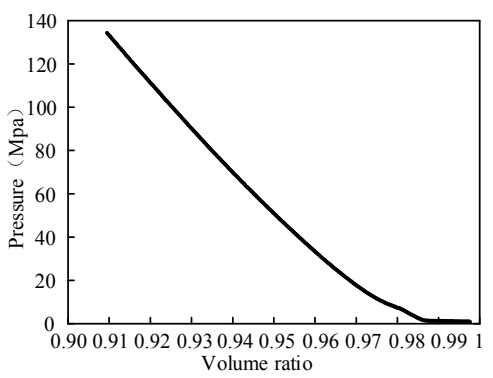

(b) Volumetric compression test data

Figure 8. Test data of the flexible joint rubber.

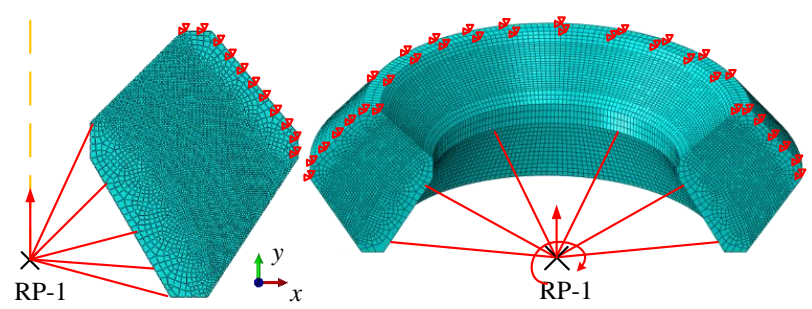

Figure 9. FE mesh of the flexible joint models.

trend of the flexible joint, but its error in the nearly linear district is bigger than the Mooney-Rivlin model and that error increases rapidly as the compression displacement increases. Thus the Mooney-Rivlin model is used to calculate the linear vertical compression stiffness of the flexible joint. According to the Mooney-Rivlin material coefficient in Table.2, the initial shear modulus is $0.706 \mathrm{MPa}$ and the Poisson's ratio is 0.4985 . The analytical, FEM and test results of the linear vertical compression stiffness of the flexjoint are respectively 291,307 and $308 \mathrm{kN} \mathrm{mm}^{-1}$. The error of the analytical result is $5.5 \%$ compared with the test result.

Figure $11 \mathrm{~b}$ shows the results of the rotation simulation and test. It can be seen that the test curve is almost linear when the rotation angle is smaller than $2^{\circ}$. Then the rotational stiffness of flexible joint decreases. The error of the Neo Hooke model is smaller than the Mooney-Rivlin model in the nonlinear district, but they both can not reflect the decrease of the rotational stiffness. The error of the Yeoh model is bigger than the Mooney-Rivlin model in the nearly linear district. But it fits the test result very well in the non-linear district. The result of the Mooney-Rivlin has obvious difference with the test result. But it fits the test result well in the nearly linear district. Thus the Mooney-Rivlin model is used to calculate the linear rotational stiffness of the flexible joint. The analytical, FEM and test results of the linear rotational stiffness of the flexible joint are respectively 441, 468 and $457 \mathrm{Nm}$ per degree. The error of the analytical result is $3.5 \%$ compared with the test result.

After analyzing Fig. 11, it can be considered that the Mooney-Rivlin model can fit the test result very well when

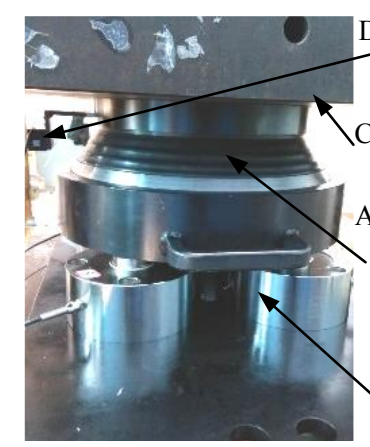

(a) Compression test

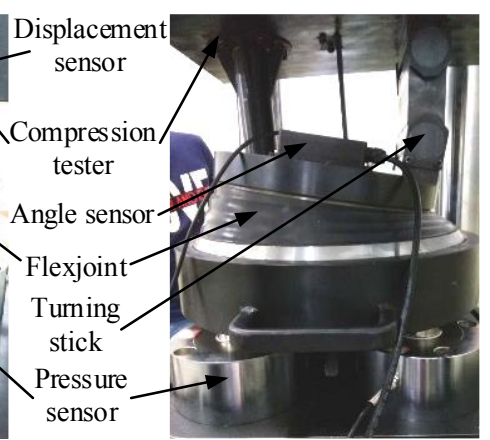

(b) Rotation test
Figure 10. The compression and rotation test device.

the rubber deformation in the flexible joint is small. The Yeoh model can reflect the deformation trend more accurately, but the error in the nearly linear district is bigger than the Mooney-Rivlin model. The analytical solutions are accurate in the nearly linear district.

The typical unfilled rubber has Poisson's ratio in the range of 0.4995 to 0.499995 and filled rubber has Poisson' ratio in the range of 0.49 to 0.497 (Hibbitt et al., 2016). In order to study the effect of Poisson's ratio on the flexible joint under compression or torsional moment, six FE models with different Poisson's ratios are created. These Poisson's ratios are $0.49,0.495,0.499,0.4995,0.4999$ and 0.49995 respectively. The corresponding incompressible parameters are $0.028,0.014,0.0028,0.0014,0.00028$ and $0.00014 \mathrm{MPa}^{-1}$.

Figure 12a shows the vertical compression stiffnesses of the flexible joints with different Poisson's ratios vs. the displacement. The analysis is taken in the nearly linear district, which means the maximum displacement is $1.5 \mathrm{~mm}$ according to Fig. 11a. The vertical compression stiffness increases by $3.45 \%$ when Poisson's ratio is 0.49 and by $8.92 \%$ when Poisson's ratio is 0.49995 . It can be found that as Poisson's ratio increases, the compression stiffness increases more considerably. Besides, as Poisson's ratio increases from 0.4999 to 0.49995 , the compression stiffness tends to be saturated.

Figure 12b shows the rotational stiffnesses of the flexible joints with different Poisson's ratios vs. the angle. The anal- 


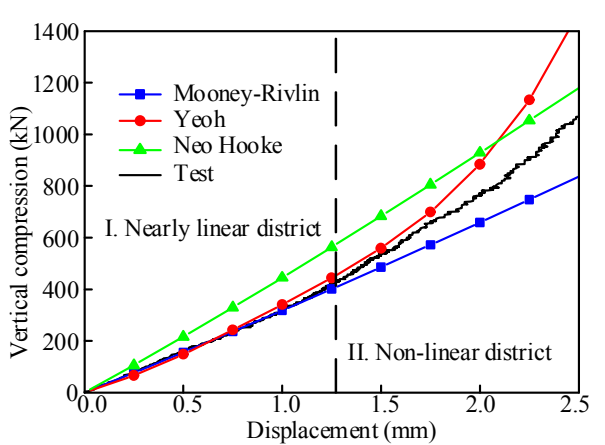

(a) Compression test and simulation

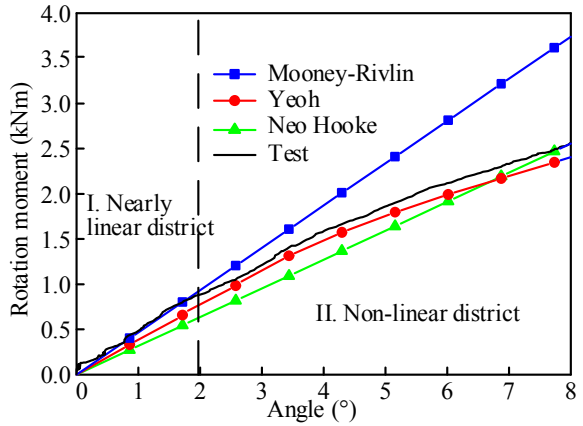

(b) Rotation test and simulation

Figure 11. The FEM simulation and test results.

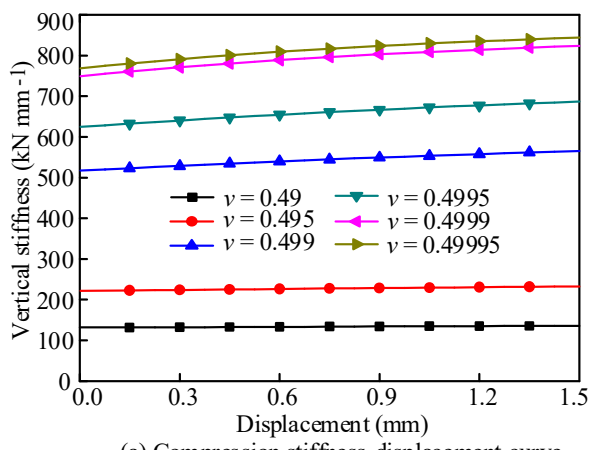

(a) Compression stiffness-displacement curve

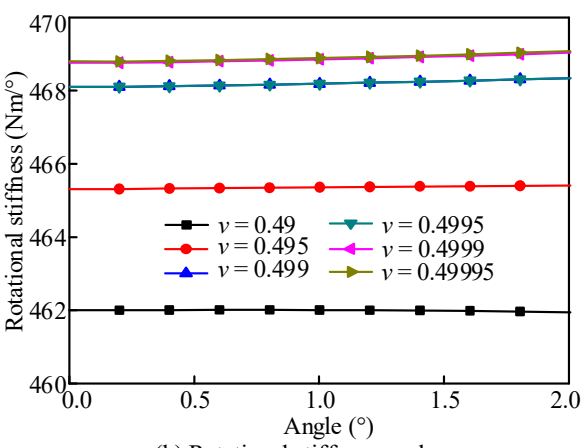

(b) Rotational stiffness-angle curve

Figure 12. Vertical/rotational stiffness variation along with vertical displacement/angle.

ysis is taken in the nearly linear district, which means the maximum angle is $2^{\circ}$ according to Fig. $11 \mathrm{~b}$. It can be seen that Poisson's ratio can barely affect the rotational stiffness. The rotational stiffness also tends to be saturated as Poisson's ratio increases from 0.4999 to 0.49995 .

By comparing two figures in Fig. 12, it can be found that Poisson's ratio has a more considerable effect on the vertical compression stiffness than the rotational stiffness. The vertical stiffness increases by 6.2 times as Poisson's ratio increases from 0.49 to 0.49995 . On the contrary, the rotational stiffness only increases by $1.47 \%$ as Poisson's ratio increases from 0.49 to 0.49995 .

\section{Conclusions}

The analytical formulae of the linear rotational stiffness are derived for the flexible joint. The rotational stiffness of rubber layer is related to the inner radius, the thickness and two edge angles. It will decrease when the inner edge angle increases and increase when the outer edge angle increases. The increase of the rubber thickness will reduce the rotational stiffness. The increase of the inner radius will enhance the rotational stiffness.

The FEM is used to verify the analytical method and analyze the stiffness of the flexible joint. The Mooney-Rivlin,
Neo Hooke and Yeoh constitutive models are used in the simulation. The experiment is taken to obtain the material coefficient and validate the analytical and simulation results. The Yeoh model can reflect the deformation trend more accurately, but the error in the nearly linear district is bigger than the Mooney-Rivlin model. The Mooney-Rivlin model can fit the test result very well when the rubber deformation in the flexible joint is small. The error of two analytical solutions are respectively 5.5 and $3.5 \%$. That's usable in the flexible joint design. The increase of Poisson's ratio of the rubber layers will enhance the vertical compression stiffness but barely have effect on the rotational stiffness. The vertical stiffness increases by 6.2 times and the rotational stiffness only increases by $1.47 \%$ as Poisson's ratio increases from 0.49 to 0.49995 .

Data availability. The data generated during this study are available from the corresponding author on reasonable request.

Competing interests. The authors declare that they have no conflict of interest. 
Acknowledgements. This research is supported by the National Natural Science Foundation of China (No. 51305088).

Edited by: Lotfi Romdhane

Reviewed by: two anonymous referees

\section{References}

Chalhoub, M. S. and Kelly, J. M.: Effect of bulk compressibility on the stiffness of cylindrical base isolation bearings, Int. J. Solids. Struct., 26, 743-760, 1990.

Chalhoub, M. S. and Kelly, J. M.: Analysis of infinite-strip-shaped base isolator with elastomer bulk compression, J. Eng. Mech., 117, 1791-1805, 1991.

Chen, G. S. and Yang, Y.: Stiffness design, simulation and test of laminated spherical elastomeric bearing, Hangkong Dongli Xuebao/journal of Aerospace Power, 30, 1512-1519, 2015.

Crocker, L. and Duncan, B.: Measurement Methods for Obtaining Volumetric Coefficients for Hyperelastic Modelling of Flexible Adhesives, National Physical Laboratory, Teddington, UK, 2001.

Donguy, P.: Development of a helicopter rotor hub elastomeric bearing, J. Aircraft, 17, 346-350, 2015.

Gent, A. N. and Lindley, P. B.: The compression of bonded rubber blocks, P. Inst. Mech. Eng., 173, 111-122, 1959.

Gunderson, R., Stevenson, A., Harris, J., Gahagan, P., and Chilton, T.: Fatigue life of TLP flexelements, Offshore Technology Conference, 4-7 May 1992, Houston, Texas, USA, 1992.

He, W. F., Liu, W. G., Yang, Q. R., and Feng, D. M.: Nonlinear rotation and shear stiffness theory and experiment research on rubber isolators, J. Eng. Mech., 138, 441-449, https://doi.org/10.1061/(ASCE)EM.1943-7889.0000350, 2012.

Hibbitt, H., Karlsson, B., and Sorensen, P.: Abaqus analysis user's manual, Dassault Systèmes Simulia Corp, Providence, USA, 2016.

Horton, J. M. and Tupholme, G. E.: Stiffness of spherical bonded rubber bush mountings, Int. J. Solids. Struct., 42, 3289-3297, https://doi.org/10.1016/j.ijsolstr.2004.10.017, 2005.
Kelly, J. M.: Earthquake-Resistant Design with Rubber, Alden Press, Oxford, England, 1993.

Kumar, A.: Performance-related parameters of elastomeric bearings, 9983 261, The University of Texas at Austin, Ann Arbor, 258 pp., 2000.

Kumar, A. E., Murthy, V. B., Mohan, R. C., and Prakash, D.: Study of Non-Linear Static Behavior of Flex Seal of Rocket Nozzle by Varying Number of Shims, Mater. Today-Proc., 2, 1613-1621, 2015.

Lampani, L., Angelini, F., Bernabei, M., Marocco, R., Fabrizi, M., and Gaundenzi, P.: Finite element analysis of a solid booster flexible bearing joint for thrust vector control, Aerotecnica \& Spazio, J. Aerospace Sci. Technol. Syst., 91, 53-61, 2012.

Landau, L. D. and Lifshitz, E. M.: Theory of Elasticity, Pergamon Print, Oxford, England, 1986.

Mishra, H. K. and Igarashi, A.: Lateral deformation capacity and stability of layer-bonded scrap tire rubber pad isolators under combined compressive and shear loading, Struct. Eng. Mech., 48, 479-500, https://doi.org/10.12989/sem.2013.48.4.479, 2013.

Stevenson, A. and Harris, J.: Fatigue life estimation of flexelements for TLP structures, in: Proceedings of the International Conference on Offshore Mechanics and Arctic Engineering, 712 June 1992, Calgary, Alberta, Canada, 241-249, 1992.

Tsai, H.-C.: Compression behavior of annular elastic layers bonded between rigid plates, J. Mech., 28, 657-663, 2012.

Tsai, H.-C. and Lee, C.-C.: Compressive stiffness of elastic layers bonded between rigid plates, Int. J. Solids. Struct., 35, 30533069, 1998.

Wang, L., Li, S., Yao, S., Lv, D., and Jia, P.: Study on the vertical stiffness of the spherical elastic layer bonded between rigid surfaces, Arch. Appl. Mech., 87, 1243-1253, https://doi.org/10.1007/s00419-017-1246-9, 2017.

Zhang, X., Liu, Y., Ren, J., and Zhan, K.: Nonlinear finite element analysis of the SRM flexible joint, in: 53rd AIAA/ASME/ASCE/AHS/ASC Structures, Structural Dynamics and Materials Conference, 20th AIAA/ASME/AHS Adaptive Structures Conference, 14th AIAA, 23-26 April 2012, Honolulu, HI, USA, 301-318, 2012. 